\title{
Optical connections on flexible substrates
}

\author{
Erwin Bosman
}

\author{
Supervisor(s): Prof. Dr. Ir. Peter Van Daele ${ }^{(1)}$, Prof. Dr. Ir. Jan Van Fleteren ${ }^{(2)}$ \\ ${ }^{(1)}$ Ghent University - Department INTEC, ${ }^{(2)}$ Ghent University - Department ELIS \\ TFCG-Microsystems, Technologiepark Building 914A, 9052 Zwijnaarde
}

\begin{abstract}
Optical interconnections integrated on a flexible substrate combine the advantages of optical data transmissions (high bandwidth, no electromagnetic disturbance, low power consumption) and those of flexible substrates (compact, ease of assembly,...). Especially the flexible character of the substrates can significantly lower the assembly cost and leads to more compact modules.

Especially in automotive-, avionic-, biomedical and sensing applications there is a great potential for these flexible optical interconnections because of the increasing data-rates, increasing use of optical sensors and requirement for smaller size and weight.

The research concentrates on the integration of commercially available polymer optical layers (SU-8, Truemode, Ormocer) on a flexible Polyimide film, the fabrication of waveguides and outof plane deflecting $45^{\circ}$ mirrors, the characterization of the optical losses due to the bending of the substrate, and the fabrication of a proof-of-principal demonstrator. The resulting optical structures should be compatible with the standard fabrication of flexible printed circuit boards.
\end{abstract}

Keywords - Optical, interconnection, flexible, waveguide, mirror.

\section{COMBINING OPTICAL AND FLEXIBLE TECHNOLOGIES.}

Optical data transmission is nowadays very common over long distances, but the ever increasing speed, density and integration of electronic components on boards forces the market to integrate optical interconnections for short distance systems as well, integrated on the boards.

The search for cheaper and smaller solutions in electronics for consumer-products has also increased the need for flexible substrates and circuits, which are lighter, more reliable and assembly-efficient.

Both increasing trends and needs result in applications which combines the advantages of optical interconnections and flexible substrate technologies. These will be realized by the integration of optical layers and components on a flexible substrate.

This paper focuses on the research on applying commercially available optical layers on a Polyimide foil or on a spin-coated Polyimide film, followed by the definition of waveguides, micro-mirrors, micro-lenses by photolithography or laserablation and aligning structures in these layers, resulting in a proof-of-principal demonstrator. Special care will be taken to ensure the compatibility of this process with the standard PCB production processes.

E. Bosman is a PhD student in the OTE Group of the INTEC Department within the TFCG Microsystems Group of the Ghent University, Belgium. Email: Erwin.Bosman@INTEC.UGent.be.

\section{INTEGRATION OF OPTICAL LAYERS ON A FLEXIBLE SUBSTRATE}

Optical layers (undercladding-, core- and uppercladdinglayer) have been successfully deposited on rigid substrates in the past by the TFCG Microsystems-group and optical elements were integrated. These layers require materials with low light propagation losses for the common wavelengths for data communication (850 $\mathrm{nm})$, telecommunication (1.3 or $1.55 \mu \mathrm{m})$ or optical sensing, depending on the application. To achieve uniformity in thickness, spincoating is used to deposit these UV-sensitive materials. Successfully applied optical layers so far were produced with Ormocer's, Truemode Backplane Polymer and SU-8.

The material for the flexible substrate itself is Polyimide (PI) which is the dominant material in the flexible circuits industry because of its good electrical, chemical, temperature and mechanical behavior. The flexible substrate needs chemical or physical surface treatment before applying the optical layers because of the inertness of Polyimide (PI). Several techniques like sandblasting and plasma etching have proven their efficiency on improving the adhesion between both the PI and the optical materials.

Working with flexible substrates demands adjustments of the process flow and special care keeping the substrate from curling up which disables proper alignment in next process steps.

Most important and crucial objective is the research of the dynamic and flexible behavior of the different optical materials, concerning the maximum bending radius and continuous bending lifetime. First tests on the SU-8, Truemode and Ormocer's materials show nice flexible behavior with maximum bending radius of $0.5 \mathrm{~cm}$ and less and good adhesion with the PI substrate after surface treatment.

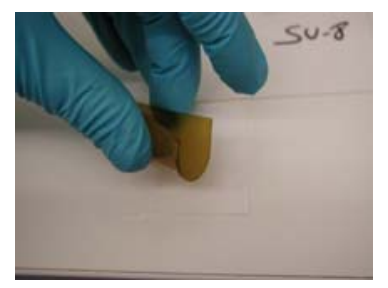

Figure 1 Upper-, core- and uppercladding of $50 \mu \mathrm{m}$ thickness each, SU-8 polymer on a $25 \mu \mathrm{m}$ Polyimide foil, bended with bending radius $0.5 \mathrm{~cm}$. ( $5 \mathrm{~cm} \times 5 \mathrm{~cm}$ substrate). 


\section{FABRICATION OF OPTICAL WAVEGUIDES AND MICRO- STRUCTURES}

\section{A. Fabrication of optical waveguides}

Light that will be coupled into the core layer will be guided in that layer due to total internal reflection, caused by the different refractive indexes of the core- and the cladding layers. Isolating tracks in the core-layer and consequently surrounding the track completely with cladding material, results in the creation of optical waveguides. This isolation can be done by patterning the core-layer with a standard photolithographic process with selective exposure of the waveguides to UV-light and in an alternative way by laserablation, removing the core-material at both sides of the waveguide.

In the past these waveguides in optical layers have been successfully applied on rigid substrates, resulting in optical propagation losses smaller than $3 \mathrm{~dB}$ per cm for a 50 x $50 \mu \mathrm{m}$ waveguide.

\section{B. Fabrication of 45 degrees out of plane turning mirror}

The data-carrying light can be vertically coupled in- and out of the waveguides with 45 degrees out of plane deflecting micro-mirrors, terminating the waveguides and connecting them with laser diodes, receivers, optical fibers, open air or optical elements. These mirrors can be realized by laserablation with a Kr-F Excimerlaser (248 nm wavelength) resulting in high performance micro-mirrors with less then $1 \mathrm{~dB}$ coupling loss and less than $100 \mathrm{~nm}$ rms surface roughness. Metallization of the mirror surface afterwards results in better reflectivity of the micro-mirror.
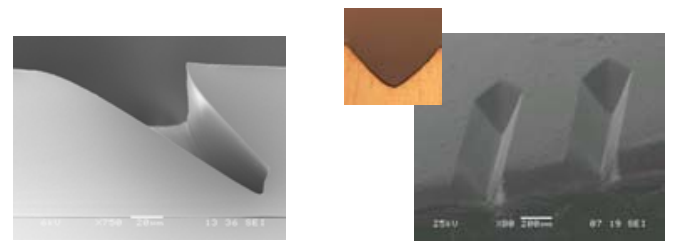

Figure 3 Laser ablated micro-mirrors in Ormocer's polymer, using a $248 \mathrm{~nm}$ wavelength Kr-F Excimer laser. Left: Tilted laser-optics result in 45 degrees tilted laserbeam-impact, Right: Adjusted mask results in a distributed impact of the laserbeam.

\section{Fabrication of alignment structures for a proof-of- principle demonstrator}

This research not only focuses on the optical assembly of mechanical circuits but the combination of optical and electronic assembly on the same module. This means that the positioning of the VCSEL's and receivers on one hand and optical fiber-arrays, optical elements and deflecting micromirrors on the other hand is crucial. Every element needs its own alignment structure, which however can all be realized with the laser ablation technique. Alignment structures consist of mechanical stand-off's adjusting the lateral position and height of an element (e.g. VCSEL, receiver), aligning holes combining lateral positioning and mechanical connection, and optical micro-lenses focusing the lightbeam into the core of another waveguide or optical fiber increasing the alignment tolerances. In concern of the standardization of this research for its applications, connections with a standard MT-ferrule will be established, using aligning holes and a lens-array. (see Figure 4)
A proof-of-principle demonstrator will be fabricated, including all the elements which have been discussed in this paper (flexible substrate, optical layers, waveguides, turning mirrors, mechanical stand-off's, lensplates, MT-ferruleconnection, VCSEL's, receivers, electrical modulator). Figure 4 shows the cross-section and principle of the demonstrator.

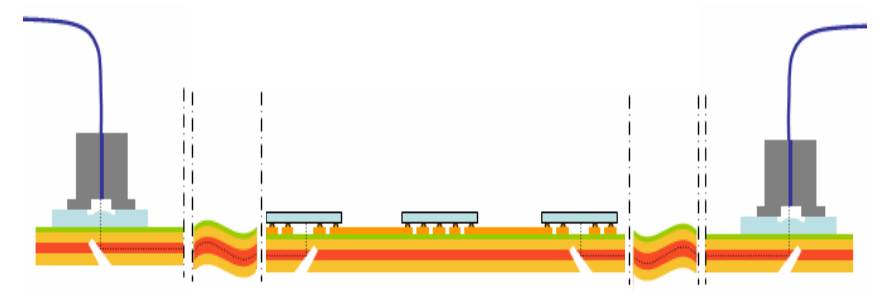

Figure 4 Cross-section of the proof-of-principle demonstrator: from left to right: MT-ferrule connection with lensplate and micromirror, flexible waveguide, VCSEL, modulator, receiver, flexible waveguide and MT-ferrule connection with lensplate and micromirror.

\section{Measurement and Characterization of propagation- and coupling losses}

Besides the static and dynamic mechanical characterization of the optical layers and the aligning structures, there is a need for optical measurements to characterize the optical path.

The emitted light of a laserdiode will be measured for its intensity and will be coupled into an optical fiber. This fiber can be positioned with a resolution of $0.5 \mu \mathrm{m}$ and can couple the light into the waveguide. At the other side of the optical path that needs to be characterized, another optical fiber can be positioned, which leads the lightbeam towards a detector. The degradation of the light intensity shows us the propagation- and coupling losses of the measured optical path.

\section{CONCLUSION}

The increasing need for flexible modules and the integration of photonics on boards results in a challenging and competitive research which combines both needs by integrating optical interconnection layers and devices on flexible substrates.

\section{ACKNOWLEDGEMENTS}

Part of this work has been carried out in the framework of the project "Plastic for Photonics" GBOU project of the IWT.

\section{REFERENCES}

[1] Joseph Fjelstad, “An engineer's guide to flexible circuit technology”, Elektromechanical Publications LTD, 1997Helmut Kopka, LaTeX, eine einführung, Addison-Wesley, 1989.

[2] G. Van Steenberge, P. Geerinck, S. Van Put, J. Van Koetsem, H. Ottevaere, D. Morlion, H. Thienpont, P. Van Daele "MT-Compatible Laser-Ablated Interconnections for Optical Printed Circuit Boards", Journal Of Lightwave Technology, Vol. 22, No. 9, September 2004, to be published

[3] http://domino.watson.ibm.com/library/CyberDig.nsf/ 0/3651a9d5eba9a25e85256e7800462108?OpenDocument

[4] Streppel U, Dannberg P, Wachter C, Brauer A, Frohlich L, Houbertz R, Popall M, "New wafer-scale fabrication method for stacked optical waveguide interconnects and 3D micro-optic structures using photo responsive (inorganic-organic hybrid) polymers”, Optical Materials, Vol. 21, 2002, pp. 475-483 